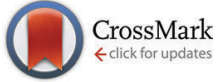

Cite this: Nanoscale Horiz., 2016, 1,60

Received 29th July 2015,

Accepted 20th November 2015

DOI: $10.1039 / c 5 n h 00036 j$

rsc.li/nanoscale-horizons

\section{Mixed-monolayer glyconanoparticles for the detection of cholera toxin by surface enhanced Raman spectroscopy $\dagger$}

\author{
Jonathan Simpson, ${ }^{\mathrm{a}}$ Derek Craig, ${ }^{\mathrm{b}}$ Karen Faulds ${ }^{\mathrm{a}}$ and Duncan Graham ${ }^{{ }^{\mathrm{a}}}$
}

We have produced silver glyconanoparticles for the sensitive (56 $\mathrm{ng} \mathrm{mL} \mathrm{mL}^{-1}$ ), low volume and rapid detection of cholera toxin $\mathrm{B}$-subunit (CTB) in synthetic freshwater (simulating the ion compositions of natural waters in which CTB could be found). This is achieved by monitoring the changes in surface enhanced Raman scattering (SERS) intensity of a Raman reporter bound to the glyconanoparticle surface. The particles selectively aggregate upon interaction with CTB, causing an increase in the measured SERS signal. The particles are designed to mimic the interactions involving the cell surface GM1 ganglioside and CTB. This is achieved by using a combination of polyethylene glycol linkers terminated with either galactose or sialic acid.

Nanoparticles have many potential applications in healthcare, including improved drug delivery and biodetection. ${ }^{1-3}$ Selective and sensitive bio-detectors can be produced by functionalising metallic nanoparticles with biomolecules such as DNA or antibodies. ${ }^{4}$ Gold and silver nanoparticles functionalised in this way, bind complementary target biomolecules and assemble to form aggregates which is indicated by the appearance of plasmoniccoupling bands in the extinction spectrum. ${ }^{5-8}$ In addition to biomolecules, Raman active molecules can be coated onto the particles giving rise to surface enhanced Raman scattering. ${ }^{9,10}$ Target-mediated aggregation of the nanoparticles creates interparticle hot spots of electromagnetic energy which further enhances the Raman signals of the surface bound Raman reporter. ${ }^{11}$ SERS sensitivity rivals that of UV-visible extinction and fluorescence spectroscopy. ${ }^{12,13}$ Additionally, the molecularly specific Raman signals can allow for multiplexed detection by using multiple Raman reporters which is important for the analysis of multianalyte samples. ${ }^{14-16}$ SERS nanoparticle biosensing has been applied to the detection of biomolecules (including DNA and antigens) along with the imaging and mapping of cells and their

${ }^{a}$ Centre for Molecular Nanometrology, Department of Pure and Applied Chemistry, University of Strathclyde (Technology and Innovation Centre), 99 George Street, Glasgow, G1 1RD, UK. E-mail: duncan.graham@strath.ac.uk

${ }^{b}$ Optical Manipulation Group, SUPA, University of St. Andrews, St. Andrews, KY16 9SS, UK

$\dagger$ Electronic supplementary information (ESI) available. See DOI: 10.1039/c5nh00036j

\section{Conceptual insights}

Cholera affects 3-5 million people each year, resulting in 100 000-120 000 reported deaths (excluding disasters). Effective water treatment relies on the ability to detect pathogens rapidly and sensitively, allowing a contaminated body of water to be isolated from local consumption promptly. Our newly created nanoparticle sensor can detect the cholera biomarker, cholera toxin B subunit (CTB), sensitively, rapidly and in low volume samples of synthetic freshwater. We demonstrate the first use of mixed carbohydrate monolayers for the rational design of carbohydrate-coated nanoparticles (glyconanoparticles) which effectively mimic the host's intestinal cell surface glycan (GM1). This subsequently allows selective interaction with the target protein, СTB. This research illustrates the first use of glyconanoparticles for the detection of СТВ by surface enhanced Raman spectroscopy (SERS). The work presents significant developments in the understanding of multivalent carbohydrate-protein interactions and in the use of SERS-active glyconanoparticles for the detection of a bacterial toxin in samples which mimic natural bodies of water.

associated substructures. ${ }^{13,15,17}$ In addition to oligonucleotides and proteins, nanoparticles can be functionalised with carbohydrates and associated species for use in glyconanotechnology. This includes enzyme inhibition, oncology and the detection of lectins (carbohydrate binding proteins) or toxins related to disease. ${ }^{18-21}$ Carbohydrates, present extensively on cell surfaces, provide multifunctional binding sites involved in crucial endogenous processes as well as in pathogen establishment. ${ }^{22-24}$

Vibrio cholerae bacteria express lectins as $\mathrm{AB}$ toxins on the pili which are key to bacterial establishment and subsequent infection. ${ }^{24}$ The B subunit (СТВ) is multifunctional, binding simultaneously to both the galactose and sialic acid moieties of the ganglioside, GM1, expressed on the surface of intestinal cells. ${ }^{24}$ Unlike the interaction between DNA base pairs or a peptide and protein, those between toxins and their related gangliosides rely on multiple unique species to stabilise the binding pocket simultaneously. ${ }^{19}$ The individually weak carbohydrate-protein interactions are strengthened by this multivalency. ${ }^{25,26}$

The mixed-carbohydrate particles described herein, mimic these extensive and complex interactions and have enabled the 
generation of glyconanoparticles which detect the CTB bacterial target both selectively and sensitively and in samples which simulate the environment in which the toxin could be found, namely synthetic freshwater samples which contain the ionic components of natural bodies of water. The approach for generating CTB-reactive particles involved deconstruction of the GM1 ganglioside to identify the major components in CTB binding. Galactose is a major component of the binding between the toxin and GM1 (see Fig. 1A), however, alone this interaction is weak (Gal-CTB $\left.K_{\mathrm{d}}=52 \mathrm{mM}\right){ }^{26,27}$ By simultaneously binding sialic acid in a symbiotic interaction, as is the case for GM1, the binding pocket is significantly stabilised (GM1-CTB $\left.K_{\mathrm{d}}=0.3 \mathrm{nM}\right){ }^{24}$

Previous research on CTB-reactive glyconanoparticles includes the development of lactose-coated gold nanoparticles for the detection of CTB at $54 \mathrm{nM}\left(3 \mu \mathrm{g} \mathrm{ml}{ }^{-1}\right) .{ }^{18}$ Our novel, mixed-monolayer galacto-sialonanoparticles (GSNPs) allow for detection of CTB with greater sensitivity than previously achieved because of the GM1-mimicking surface and by using SERS for detection.

$50 \mathrm{~nm}$ colloidal silver nanoparticles, prepared using ethylenediaminetetraacetic acid (EDTA) reduction, were used to produce the biosensor. The Raman molecule used in analysis was the benzotriazole dye molecule 4 - $((1 H$-benzo[ $[d][1,2,3]$ triazol-6-yl)diazenyl)-3,5-dimethoxyphenol (RB1) previously prepared by our

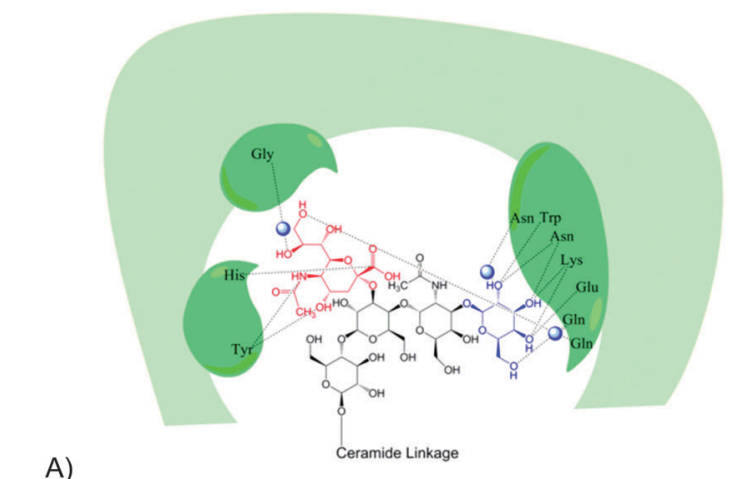

A)

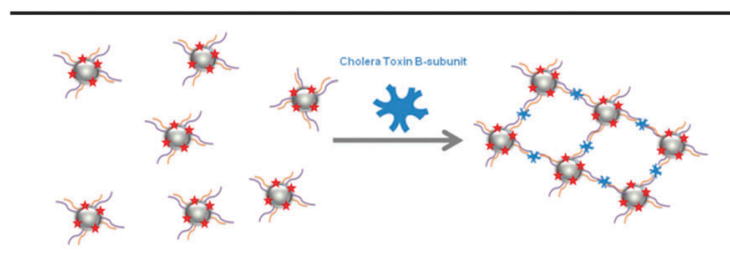

B)

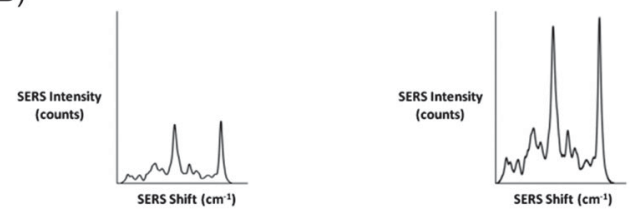

Fig. 1 (A) GM1-CTB binding pocket structure (H-bonding) with sialic acid (red) and galactose (blue) residues shown. Water is shown as blue spheres. (B) Galactose and sialic acid linkers (orange and purple chains respectively) are used to coat the nanoparticles prior to incubation with CTB. The close proximity of the nanoparticles following aggregation with CTB results in "hot-spots" of electromagnetic energy which increase the scattering intensity of surface bound RB1 (red stars) SERS signal. group. ${ }^{28}$ The carbohydrates were subsequently tethered to the nanoparticle surface via thiolated polyethylene glycol (PEG) linkers. PEG provides steric protection that helps discourage aggregation by increasing the minimum interparticle distance in the colloid. Additionally, non-specific binding of biological or chemical species present in a sample is reduced. PEGylated galactose and sialic acid were produced by amide coupling (see ESI, $\dagger$ Section 6 and Fig. S2 for details) and used to functionalise the RB1-coated silver nanoparticles with GM1 mimics.

The SERS-active GSNPs were characterised by UV-visible extinction spectroscopy, dynamic light scattering and gel electrophoresis (see ESI, $\dagger$ Table S4 and Fig. S3). The surface coverage-activity relationship was evaluated by functionalising the particles with galactose and sialic acid linkers of different lengths and altering the ratio between the linkers. While the PEG coating, together with size of CTB, leaves space between the particles upon CTB binding, plasmonic coupling has been shown to occur up to a distance of $70 \mathrm{~nm} \cdot{ }^{29,30}$ It was therefore proposed that upon aggregation of GSNPs with CTB, an increased SERS response would be measured. The GSNPs were incubated with $80 \mathrm{nM}$ CTB for 5 minutes and the resulting aggregation measured by monitoring changes in SERS intensity of the RB1 peak at $1364 \mathrm{~cm}^{-1}$ (see Fig. 1B).

The importance of both sialic acid and galactose in binding GM1 is demonstrated in Fig. 2. The greatest SERS intensity change for the glyconanoparticles is noted with the mixed galactose/sialic acid (15:1) monolayer. There is little interaction and hence low SERS increase when using sialic acid or galactose as the sole surface coating.

Our investigations reveal a maximum normalised SERS enhancement of 5.2 at a PEGylated carbohydrate ratio (galactose to sialic acid) of 15:1 (see Table 1), where the two carbohydrate linker types are of different lengths ( $\mathrm{PEG}_{12} \mathrm{Gal}$ and $\left.\mathrm{PEG}_{18} \mathrm{Sia}\right)$. The extension of the sialic acid from the nanoparticle surface with respect to the galactose encourages maximal binding and hence aggregation. This arises from the minimal steric interference from surrounding galactose molecules with sialic acid. The reduced amount of sialic acid on the surface with respect to the galactose indicates the importance of isolated galactose/ sialic acid-CTB interactions. The interaction, aggregation and hence SERS enhancement is reduced when the sialic acid is

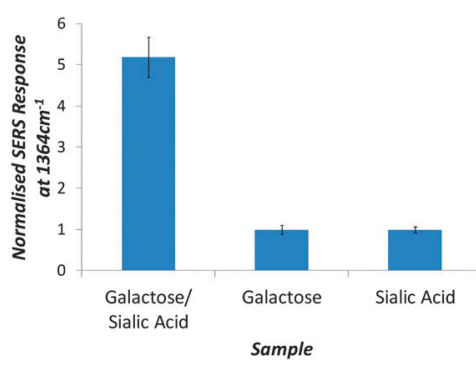

Fig. 2 Normalised SERS response for mixed or single carbohydrate-coated nanoparticles 5 minutes after addition of 80 nM CTB in HEPES buffer, $\mathrm{pH}$ 7.4. Those particles coated uniquely in either sugar are incubated with $30 \mu \mathrm{M}$ of the appropriate PEGylated sugar prior to testing with CTB. The laser excitation wavelength used is $514.5 \mathrm{~nm}$ with a 1 second acquisition, 3 accumulations and 3 replicates measured. 
Table 1 CTB-mediated SERS enhancement with different galacto-sialo surface ratios and PEG chain lengths. The normalised SERS enhancement values are listed below the corresponding galactose to sialic acid ratio ${ }^{a}$

\begin{tabular}{lllll}
\hline & \multicolumn{4}{l}{ Normalised SERS enhancement at $1364 \mathrm{~cm}^{-1}$} \\
\cline { 2 - 5 } Coverage type & $1: 1^{a}$ & $3: 1^{a}$ & $15: 1^{a}$ & $30: 1^{a}$ \\
\hline $\mathrm{PEG}_{12} \mathrm{Gal} / \mathrm{PEG}_{12} \mathrm{Sia}$ & $1.2( \pm 0.16)$ & $2.0( \pm 0.20)$ & $3.5( \pm 0.19)$ & $3.0( \pm 0.21)$ \\
$\mathrm{PEG}_{12} \mathrm{Gal} / \mathrm{PEG}_{18} \mathrm{Sia}$ & $0.9( \pm 0.10)$ & $1.7( \pm 0.22)$ & $5.2( \pm 0.49)$ & $3.3( \pm 0.51)$ \\
$\mathrm{PEG}_{18} \mathrm{Gal} / \mathrm{PEG}_{12} \mathrm{Sia}$ & $0.9( \pm 0.06)$ & $0.9( \pm 0.09)$ & $0.9( \pm 0.06)$ & $1.0( \pm 0.07)$ \\
$\mathrm{PEG}_{18} \mathrm{Gal} / \mathrm{PEG}_{18} \mathrm{Sia}$ & $0.9( \pm 0.20)$ & $1.7( \pm 0.15)$ & $1.8( \pm 0.07)$ & $2.3( \pm 0.14)$ \\
& & \\
${ }^{a}$ The enhancement is a ratio of the RB1 signal at $1364 \mathrm{~cm}^{-1}$ prior to \\
aggregation with $80 \mathrm{nM} \mathrm{CTB}$ to the same signal measured 5 minutes \\
after CTB addition in HEPES buffer, pH 7.4. The laser excitation \\
wavelength used is 514.5 nm with a 1 second acquisition, 3 accumula- \\
tions and 3 replicates measured.
\end{tabular}

extended from the nanoparticle surface at an equivalent length to the galactose. While nanoparticles provide a multivalent platform that can strengthen individually weak carbohydrate-lectin/toxin interactions, they can also hinder binding if the surface coating is too crowded. Binding is discouraged as the spacing between repeating galactose and sialic acid pairs may not match the toxin shape or space between the toxin binding units. Additionally, closely-packed carbohydrates may hydrogen bond, reducing protein-binding. This provides an explanation for the reduced SERS enhancement in both the case of the $\mathrm{PEG}_{12} \mathrm{Gal} / \mathrm{PEG}_{12} \mathrm{Sia}$ and $\mathrm{PEG}_{18} \mathrm{Gal} / \mathrm{PEG}_{18} \mathrm{Sia}$. Additionally in the case of the later, the increase in length of both PEG chains increases the distance between aggregated nanoparticles. The magnitude of SERS enhancement is distance dependent and increasing the interparticle distance with the longer PEG chains generates weaker hot spots, resulting in a lower enhancement compared with those particles coated with the shorter $\mathrm{PEG}_{12} \mathrm{Gal}$.

The selectivity of the particles is demonstrated in Fig. 3. Incubating the GSNPs with $100 \mathrm{nM}$ of the glucose-specific plant lectin, Concanavalin A (which interacts specifically with gluconanoparticles, see ESI, $\dagger$ Fig. S6) causes no aggregation and hence gives no appreciable change in SERS intensity even after 30 minutes of incubation. The СTB limit of detection $(1 \mathrm{nM}$ or $56 \mathrm{ng} \mathrm{mL} \mathrm{m}^{-1}$ ) achieved by using the GSNPs is 50 times greater than that achievable by UV-visible extinction spectroscopy (54 $\mathrm{nM}$ or $\left.3 \mu \mathrm{g} \mathrm{mL}{ }^{-1}\right) \cdot{ }^{18}$ This falls within the recommended detection range of $10 \mathrm{pg} \mathrm{mL}^{-1}$ to $100 \mathrm{ng} \mathrm{mL}^{-1}$ and matches the limits of WHO approved tests including infant rabbit and coagglutination assays, demonstrating the relevancy of this detection level. ${ }^{31,32}$

The mixed-monolayer glyconanoparticles presented herein react rapidly with СТВ in a selective and sensitive manner, giving results in 5 minutes. A low volume is required to aggregate the nanoparticles (10 $\mu \mathrm{L}$ CTB sample in $200 \mu \mathrm{L}$ of sensor). Previous research has demonstrated that CTB can be measured in both patient and water samples, however this involves PCR or antibody-based methods with multiple assay steps required for eventual detection. ${ }^{33,34}$ The capability of the rapid and sensitive nanoparticle sensor described herein has been demonstrated in synthetic freshwater samples. By developing our proof-of-concept, we aim to generate a working CTB assay for use in patient samples. Additionally, the availability of portable Raman spectrometers could allow for field

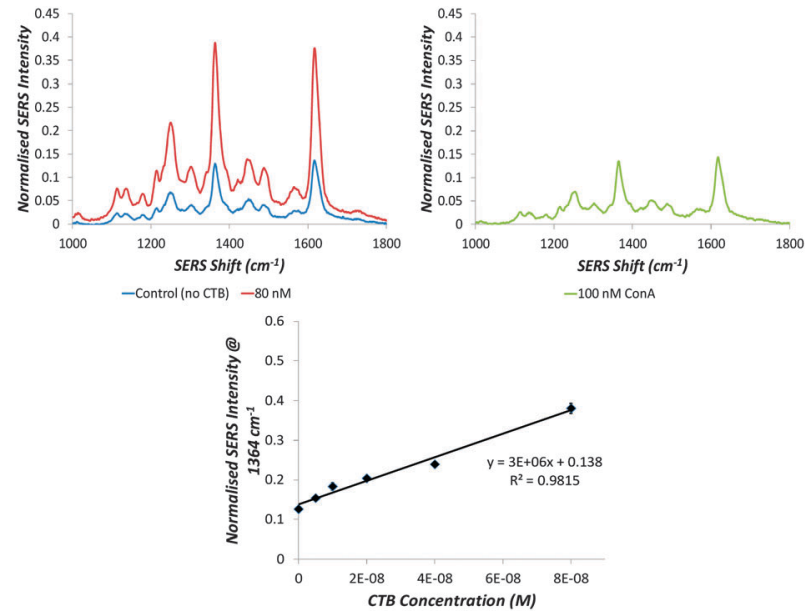

Fig. 3 RB1 SERS spectra of GSNPs before and after addition of (top left) CTB or (top right) ConA in synthetic freshwater. (Lower middle) Normalised SERS Intensity of $1364 \mathrm{~cm}^{-1}$ peak measured 5 minutes after addition of the indicated concentration of CTB with 5 replicates per concentration. The limit of detection determined is $1 \mathrm{nM}$. The laser excitation wavelength used is $514.5 \mathrm{~nm}$ with a 1 second acquisition and 3 accumulations, 5 replicates measured.

testing with the GSNPs. A similar preparation strategy could be employed to generate particles which mimic the G1b gangliosides (GT1b, GD1b and GQ1b) for the detection of tetanus and botulism toxins by SERS.

The authors acknowledge the Carnegie Trust for the Universities of Scotland for funding. DG acknowledges support from the Royal Society through a Wolfson Research Merit Award.

\section{Notes and references}

1 C. R. Steven, G. A. Busby, C. Mather, B. Tariq, M. L. Briuglia, D. A. Lamprou, A. J. Urquhart, M. H. Grant and S. V. Patwardhan, J. Mater. Chem. B, 2014, 2, 5028-5042.

2 R. Rojanathanes, A. Sereemaspun, N. Pimpha, V. Buasorn, P. Ekawong and V. Wiwanitkit, Taiwan. J. Obstet. Gynecol., 2008, 47, 296-299.

3 L. E. Jamieson, A. Jaworska, J. Jiang, M. Baranska, D. J. Harrison and C. J. Campbell, Analyst, 2015, 140, 2330-2335.

4 A. Lesniewski, M. Los, M. Jonsson-Niedziolka, A. Krajewska, K. Szot, J. M. Los and J. Niedziolka-Jonsson, Bioconjugate Chem., 2014, 25, 644-648.

5 C. A. Mirkin, R. L. Letsinger, R. C. Mucic and J. J. Storhoff, Nature, 1996, 382, 607-609.

6 C. Zhang, Z. Zhang, B. Yu, J. Shi and X. Zhang, Anal. Chem., 2001, 74, 96-99.

7 D. C. Hone, A. H. Haines and D. A. Russell, Langmuir, 2003, 19, 7141-7144.

8 S. Schlücker, Angew. Chem., Int. Ed., 2014, 53, 4756-4795.

9 D. Graham, G. McAnally, J. C. Jones and W. Ewen Smith, Chem. Commun., 1998, 1187-1188.

10 K. Saha, S. S. Agasti, C. Kim, X. Li and V. M. Rotello, Chem. Rev., 2012, 112, 2739-2779.

11 G. V. P. Kumar, S. Shruthi, B. Vibha, B. A. A. Reddy, T. K. Kundu and C. Narayana, J. Phys. Chem. C, 2007, 111, 4388-4392. 
12 K. Faulds, R. P. Barbagallo, J. T. Keer, W. E. Smith and D. Graham, Analyst, 2004, 129, 567-568.

13 D. Craig, J. Simpson, K. Faulds and D. Graham, Chem. Commun., 2013, 49, 30-32.

14 E. Smith and G. Dent, Modern Raman Spectroscopy: A Practical Approach, John Wiley \& Sons Ltd., Chichester, West Sussex, 1st edn, 2005.

15 K. Faulds, F. McKenzie, W. E. Smith and D. Graham, Angew. Chem., 2007, 119, 1861-1863.

16 R. A. Halvorson and P. J. Vikesland, Environ. Sci. Technol., 2010, 44, 7749-7755.

17 K. L. Rule and P. J. Vikesland, Environ. Sci. Technol., 2009, 43, 1147-1152.

18 C. L. Schofield, R. A. Field and D. A. Russell, Anal. Chem., 2007, 79, 1356-1361.

19 N. C. Reichardt, M. Martin-Lomas and S. Penadés, Chem. Soc. Rev., 2013, 42, 4358-4376.

20 B. K. Gorityala, Z. Lu, M. L. Leow, J. Ma and X.-W. Liu, J. Am. Chem. Soc., 2012, 134, 15229-15232.

21 D. Craig, S. McAughtrie, J. Simpson, C. McCraw, K. Faulds and D. Graham, Anal. Chem., 2014, 86, 4775-4782.

22 T. K. Lindhorst, Essentials of Carbohydrate Chemistry and Biochemistry, Wiley-VCH Verlag GmbH \& Co. KGaA, Weinheim, 3rd edn, 2007.
23 L. Chen and F. Li, J. Virol., 2013, 87, 4118-4120.

24 E. A. Merritt, S. Sarfaty, F. V. D. Akker, C. L'Hoir, J. A. Martial and W. G. J. Hol, Protein Sci., 1994, 3, 166-175.

25 R. J. Pieters, Org. Biomol. Chem., 2009, 7, 2013-2025.

26 M. Bergstrom, S. Liu, K. L. Kiick and S. Ohlson, Chem. Biol. Drug Des., 2009, 73, 132-141.

27 J. Sanchez and J. Holmgren, Indian J. Med. Res., 2011, 133, 153-163.

28 G. McAnally, C. McLaughlin, R. Brown, D. C. Robson, K. Faulds, D. R. Tackley, W. E. Smith and D. Graham, Analyst, 2002, 127, 838-841.

29 C. Sonnichsen, B. M. Reinhard, J. Liphardt and A. P. Alivisatos, Nat. Biotechnol., 2005, 23, 741-745.

30 L. Guerrini, F. McKenzie, A. W. Wark, K. Faulds and D. Graham, Chem. Sci., 2012, 3, 2262-2269.

31 CDC, Centre for Disease Control and Prevention - Laboratory Testing for Cholera, 1993, pp. 62-88.

32 D. L. Evers, J. He, J. T. Mason and T. J. O'Leary, J. Clin. Microbiol., 2010, 48, 4620-4622.

33 U. Tuteja, S. Kumar, J. Shukla, J. Kingston and H. V. Batra, J. Med. Microbiol., 2007, 56, 1340-1345.

34 V. L. Schmit, R. Martoglio and K. T. Carron, Anal. Chem., 2012, 84, 4233-4236. 